Carlton Himes, Eric Carlson, Ryan J. Ricchiuti, David W. Taylor, Brian Otis and Babak A. Parviz

\title{
Using plants for directly powering nanoelectronic circuits
}

\begin{abstract}
There are varying electrical potentials throughout the structures of vascular plants and their surrounding environments resulting from the transport of charged ions. Nanotechnology allows one to tap into these potentials to create a sustainable power supply which is useable by electronic circuits. We characterized the performance of bigleaf maple (Acer macrophyllum) trees as a power supply and found voltages on the order of hundreds of millivolts. Nanotechnology has enabled the creation of ultralow voltage and ultralow power circuits that can use living trees directly as a power source. We designed and fabricated two low-power integrated circuits (ICs) for remote sensing networks and successfully powered them solely from Acer macrophyllum. The first circuit, built in a $130 \mathrm{~nm}$ CMOS process, is a boost converter generating a stable $1.1 \mathrm{~V}$ output. It consumes only 10 nanowatts when duty-cycled and it can operate on voltages as low as $20 \mathrm{mV}$. The second circuit, built in a $90 \mathrm{~nm}$ CMOS process, is a $0.045 \mathrm{~Hz}$ oscillator consuming only $2.5 \mathrm{nW}$ during constant operation. These two circuits can provide useable voltage, timing signals, and "wake-up" functionality to remote sensor nodes in environmental monitoring.
\end{abstract}

Nanotechnology Perceptions 6 (2010) 29-40

Nonsubscribers purchase individual article 